\title{
Integration Of Anesthesia Into ECT: A Hospital- Based Study On MECT From Anesthesiologist Point Of View
}

\author{
Neupane $\mathrm{S}^{1}$, Pahari $\mathrm{T}^{2}$, Lamichhane $\mathrm{N}^{3}$, Thapa $\mathrm{DK}^{3}$ \\ 1. Lecturer, Department of Anesthesia, Gandaki Medical College, Pokhara, Nepal 2 Associate Professor, \\ Department of Anesthesia, Gandaki Medical College, Pokhara, Nepal 3. Professor, Department of \\ Psychiatry, Gandaki Medical College, Pokhara, Nepal \\ E-mail *Corresponding author: ddthapa@ hotmail.com
}

\begin{abstract}
Introduction: MECT is a unique procedure where psychiatrist and anesthesiologist work together as a team. It is estimated that annually, approximately 1 million patients receive ECT worldwide. Due to association of physical complications like long bone and vertebrae fractures with direct ECT, anesthesia has been integrated into ECT. MECT is now the globally accepted standard mode of ECT. This study was done to see the Socio-demographic and clinical profile of patients receiving MECT.
\end{abstract}

Material And Method: This is a hospital-based study done in BG hospital and Research Centre, Pokhara, Nepal for the duration of 12 months; from Baisakh, 2074 to Chaitra, 2074. A total of 50 consecutives cases that received $M E C T$ were enrolled after written informed consent for the ECT from the patients' caretaker. The patient's details, diagnosis and the numbers of cycles of MECT thus applied till clinical improvement were noted during the study period. MECT was administered on alternate days. The assessment of clinical improvement was done by the consultant psychiatrist. Following the protocol, each cycle of MECT was applied by consultant psychiatrist in the presence of the consultant anesthesiologist.

Results: The total number of cases enrolled were 50. Out of 50 cases, 41 (82\%) were male and mean age was 30.60 \pm 10.13 years. The most common indications for MECT were BPAD (74\%). The number of cycles of modified MECT applied on the individuals ranged from 6 to 18 cycles. Majority of the cases (78\%) seem to have a clinical improvement after 6 cycles of modified ECT. There was no complication that warranted the termination of MECT.

Conclusion: MECT when used judiciously is safe and leads to better clinical improvement.

Keywords: MECT; psychiatric disorders; Nepal

\section{INTRODUCTION}

Modified electroconvulsive therapy (MECT) is a unique procedure where psychiatrist and anesthesiologist work together as a team. Historically, electroconvulsive therapy (ECT) was first used in Italy in $1938 .{ }^{1}$ Since then it has become an effective method of treatment of several psychiatric disorders. 2,3 But with the advent of psychotropics there was a sharp decrease in the use of ECT. However, the use of ECT began to increase again, especially during the 1970s for the treatment of patients resistant to pharmaceutical treatment. ${ }^{4}$ It is estimated that annually, approximately 1 million patients receive ECT worldwide. ${ }^{5}$

The most common indication of ECT is major depressive disorder, especially in cases who have failed medications trials, have not tolerated medications, have severe or psychotic symptoms, are suicidal or homicidal or have marked symptoms of agitation or stupor. The other common indications are mania, bipolar affective disorder (BPAD), schizophrenia etc. The ECT can also be used in obsessive compulsive disorder (OCD), delirium, neuroleptic malignant syndrome, intractable seizure, on and off phenomenon in Parkinsonism etc. ${ }^{6}$

Although guidelines for the administration of ECT have been suggested, there are marked variations in ECT standards and practice between countries and between institutions in each country. ${ }^{7}$ Survey of ECT practice in India has shown that $13.4 \%$ of psychiatrists used ECT 
during the preceding 6 months. 8,9 Approximately, $30 \%$ of psychiatrists used unmodified or direct ECT. (India 6) A 2002 study suggested that there may be an even larger usage of unmodified ECT in India. ${ }^{10}$ Physical complications like long bone and vertebrae fractures have been long associated with direct ECT. ${ }^{11}$ The integration of anesthesia into ECT seems to have been precipitated by such findings of Dewald et al. ${ }^{12}$ The MECT has been the global accepted standard. In our study, we have used the MECT.

\section{MATERIAL AND METHOD}

This is a hospital-based study done in BG hospital and Research Centre, Pokhara, Nepal for the duration of 12 months; from Baisakh, 2074 to Chaitra, 2074. The total numbers of 50 consecutives cases that received MECT were enrolled. The written consent for the ECT was taken from the patients' caretaker after the detailed clinical discussion of the case and the need for application of ECT. The patient's details, diagnosis and the numbers of cycles of MECT thus applied till clinical improvement was achieved were noted during the study period. The MECT was administered on alternate days. The assessment of clinical improvement was done by the consultant psychiatrist.

The ECT machine used for administering MECT was ECTON, manufactured by RMS,

Chandigarh, India. Brief pulse mode was used

while administering the MECT.

The followings were the protocols observed for the application of MECT. Only admitted, ASA-I patients were included. Patients undergoing MECT had pre-anesthetic assessment a day prior to the procedure from the consultant anesthesiologist. Patients were evaluated and necessary investigation were done to rule out any medical conditions. Patients were kept nil per orally for at least 8 hours before they were taken to MECT procedure room. MECT was done under general anesthesia using Propofol $(1-2 \mathrm{mg} / \mathrm{kg})$ as an inducing agent. Succinylcholine $(1.5 \mathrm{mg} / \mathrm{kg})$ was used along with the mentioned agent. Face-masked ventilation was done using $100 \%$ oxygen and vital signs were monitored. One of the arms was spared with BP cuff before administration of muscle relaxant to monitor the occurrence and duration of seizure during application of MECT. Bilateral electrodes were used. Seizure duration of at least 25 seconds was considered to be effective. In cases failing to produce effective seizure duration, upward titration of electrical dose was done. After the procedure, patient was shifted to observation room where patient was kept under oxygen with vital signs being monitoring. After they regained the consciousness, patients were shifted to the ward. Following the protocol, each cycle of MECT was applied by consultant psychiatrist in the presence of the consultant anesthesiologist.

\section{RESULT}

During the study period of 1 year, the total number of cases enrolled were 50 . Out of 50 cases, $41(82 \%)$ were male and $9(18 \%)$ were female. The age of the patients ranged from 18 to 60 years with the mean age of $30.60 \pm 10.13$ years. All the patients were literate and most were Hindu by religion.

Among the various age groups, majority of the cases, $18(36 \%)$ belonged to 26 to 30 years followed by $8(16 \%)$ in 15 to 20 and $7(14 \%)$ in 21 to 25 years of age group. Few of the cases, $4(8 \%)$ belonged to elderly age group.

Table: 1 Showing the various age groups:

\begin{tabular}{|l|l|l|}
\hline $\begin{array}{l}\text { Age group in } \\
\text { years }\end{array}$ & Number & $\begin{array}{l}\text { Percentage } \\
\mathbf{( \% )}\end{array}$ \\
\hline $15-20$ & 8 & 16 \\
\hline $21-25$ & 7 & 14 \\
\hline $26-30$ & 18 & 36 \\
\hline $31-35$ & 4 & 8 \\
\hline $36-40$ & 6 & 12 \\
\hline $41-45$ & 3 & 6 \\
\hline$\geq 46$ & 4 & 8 \\
\hline Total & 50 & 100 \\
\hline
\end{tabular}

The most common indications for MECT were BPAD (74\%) followed by schizophrenia (14\%) and Recurrent depressive disorder (RDD) and multiple substance abuse with induced psychosis, each constituting $4 \%$ of the total cases. 
Table 2: Showing the various indications of MECT:

\begin{tabular}{|l|l|l|}
\hline Indications & Number & $\begin{array}{l}\text { Percentage } \\
(\mathbf{0})\end{array}$ \\
\hline BPAD & 37 & 74 \\
\hline Schizophrenia & 7 & 14 \\
\hline RDD & 2 & 4 \\
\hline $\begin{array}{l}\text { MSA with induced } \\
\text { psychosis }\end{array}$ & 2 & 4 \\
\hline OCD & 1 & 2 \\
\hline $\begin{array}{l}\text { Seizure with } \\
\text { interictal psychosis }\end{array}$ & 1 & 2 \\
\hline Total & 50 & 100 \\
\hline
\end{tabular}

The number of cycles of modified MECT applied on the individuals ranged from 6 to 18 cycles. Majority of the cases $(78 \%)$ seem to have a clinical improvement after 6 cycles of modified ECT. The prescribed medications were continued as advised by the consultant psychiatrist in the center.

Table 3: Showing the various cycles of MECT applied for clinical improvement:

\begin{tabular}{|l|l|l|}
\hline $\begin{array}{l}\text { Number of } \\
\text { cycles of MECT }\end{array}$ & Number & $\begin{array}{l}\text { Percentage } \\
(\%)\end{array}$ \\
\hline 6 & 39 & 78 \\
\hline 8 & 4 & 8 \\
\hline 10 & 1 & 2 \\
\hline 11 & 1 & 2 \\
\hline 12 & 4 & 8 \\
\hline 18 & 1 & 2 \\
\hline Total & 50 & 100 \\
\hline
\end{tabular}

In our study, there was no complication that warranted the termination of MECT.

\section{DISCUSSION:}

ECT has proven to be an effective method of treatment in indicated psychiatric disorders. Though there had been a decline in its use after the advent of psychotropics, its use began to increase again after the emergence of drug resistant cases. ${ }^{4}$ Globally MECT is the accepted standard method of ECT.

In several studies on practice of ECT in Asians countries, it has been found that unmodified ECT is frequent, unilateral ECT is rarely administered, sine wave ECT devices are still used in many centers and electroencephalogram
(EEG) monitoring is not usual. ${ }^{13}$ In Russian federation, $94 \%$ of institutions used bifrontotemporal ECT and 39\% institutions used brief pulse device. ${ }^{14}$ In our study, we had used bilateral electrode placement, modified version of ECT and brief pulse ECT device. Brief pulse devices cause less cognitive disruption and is ideal when compared to sine wave devices. ${ }^{16}$

In our study, both the consultant psychiatrist and anesthesiologist worked together as a team and electrical stimulus during application of MECT was activated by the psychiatrist while anesthetist was engaged in monitoring the vitals of the patient. In response to who most frequently activates the ECT stimulus, a study on ECT in Canada revealed that $96 \%$ of the centers reported a psychiatrist, 3\% reported a family physician and $1 \%$ reported a psychiatry resident under the supervision of psychiatrist. 16 The activation of ECT stimulus under the supervision of psychiatrist may be a common practice in medical college throughout the country.

In the Canadian survey, it was found that $90 \%$ of the survey completers routinely used electroencephalography (EEG) to time seizure duration. 16 Based on Russian survey, it was found that $83 \%$ monitor the motor seizure by simple visual inspection on unmodified ECT or by the cuff technique with MECT where as $17 \%$ do not monitor it. ${ }^{14}$ In our study, we timed the seizure by the cuff technique over one of the arms.

When there is missed or aborted seizure, a study by Gosselin et al 16 revealed that, $89 \%$ immediately re-stimulated at higher stimulus intensity. Six percent re-stimulated at the same dose and $4 \%$ stimulated at a higher intensity in the following treatment session. In our study, in case of aborted seizure, higher stimulus intensity was immediately re-stimulated.

In our study, out of 50 cases, 41 (82\%) were male and $9(18 \%)$ were female. The age of the patients ranged from 18 to 60 years with the mean age of $30.60 \pm 10.13$ years. Among the various age groups, our study showed that majority of the cases, $18(36 \%)$ belonged to $26-$ to 30 years followed by 8 (16\%) in 15- 20 and 7 (14\%) in 2125 years of age group. Only very few of the cases belonged to elderly age group. 
Based on the gender, Australian and Japanese studies have shown the slight preponderance of female patients receiving ECT. ${ }^{17,1}$ The Nepalese study done in the country's largest psychiatric facility showed slight female preponderance at treatment with ECT. ${ }^{18}$ Our study where males were largely dominant matched with the study done at the only government mental hospital in the country. ${ }^{19}$ The dominant age group of our study matched with the same study by Sherchan and Joshi done at the government hospital. ${ }^{19}$ In our study, the number of cycles of MECT applied on the individuals ranged from 6 to 18 cycles. Majority of the cases $(78 \%)$ seem to have a clinical improvement after 6 cycles of modified ECT. In the study by Sherchan and Joshi, out of the total 64 patients, the most of the patients $(37 \%)$ received 6 cycles of ECT. ${ }^{19}$ In the Russian survey, the typical ECT course length was from 4 to 15 treatment sessions. ${ }^{14}$

The Asians study revealed schizophrenia $(41.8 \%)$, major depression (32.5\%), mania (14\%), catatonia $(6.9 \%)$ and drug abuse $(1.8 \%)$ as five the commonest indication of ECT. (13) The Indian survey on ECT showed schizophrenia (36.5\%), major depression (33.5\%), mania 917.9\%), catatonia $(6.2 \%)$ and dysthymia $(2.8 \%)$ as the five commonest indication of ECT. ${ }^{15}$ The Nepalese study done in the country's largest psychiatric facility showed schizophrenia $(44.5 \%), \quad$ BPAD/ Mania (29.6\%), severe depression with psychotic features (11.1\%), ATPD $(6.2 \%)$ etc as the commonest indications for ECT. 18 In another Nepalese study by Sherchan and Joshi the commonest indication for ECT were ATPD, schizophrenia, BPAD/ Mania and depression. ${ }^{19}$ In our study, the most common indications for MECT were BPAD (74\%) followed by schizophrenia (14\%) and Recurrent depressive disorder (RDD) and multiple substance abuse with induced psychosis, each constituting $4 \%$. In our study, there was no complication that warranted the termination of MECT. This outcome may have resulted from the careful preanesthetic measure taken before application of electroconvulsive therapy. Similarly, in the study by Nelson A I, no ECT- related death was reported and $41 \%$ of the respondents reported that complications were rare or absent. 14

Unfortunately, Psychiatry is a subject which has not been receiving its due attention and importance despite the common prevalent of mental disorders. WHO has stated that the mental health problems are on the rise and is projected to be the biggest contributing factor of morbidity in the coming decade. ECT despite being an effective form of treatment, is one of the procedures that is misinterpreted. It is often portrayed in media and movies as terrifying and more often as a form of punishment. From the anesthesiologist point of view, let me say that this article proves otherwise.

\section{CONCLUSION:}

MECT when used judiciously is safe and leads to better clinical improvement.

\section{ACKNOWLEDGEMENT: None}

\section{FUNDING: None}

\section{CONFLICT OF INTEREST: None}

\section{REFERENCES:}

1. Chanpattana $W$, Kojima K, Kramer BA, Intakorn A, Sasaki S, Kitphati R. ECT practice in Japan. The Journal of ECT. 2005 Sep 1;21(3):139-44.

2. Abrams R. Electroconvulsive therapy. Oxford University Press; 2002 Jun 27.

3. Weiner RD. The practice of electroconvulsive therapy: recommendations for treatment, training, and privileging: a task force report of the American Psychiatric Association. American Psychiatric Pub; 2001.

4. Fink $M$, Sackeim HA. Convulsive therapy in schizophrenia?. Schizophrenia Bulletin. 1996 Jan 1;22(1):27-39.

5. Prudic J, Olfson M, Sackeim HA. Electro-convulsive therapy practices in the community. Psychological medicine. 2001 Jul 1;31(5):929.

6. Sadock BJ, Sadock VA, Synopsis. Lippincott Williams and Wilkins; 2007

7. Eranti SV, McLoughlin DM. Electroconvulsive therapy: state of the art. Br J Psychiatry. 2003;182:89.

8. Agarwal AK, Andrade C, Reddy MV. The practice of $E C T$ in India: Issues relating yo the administration of ECT. Indian journal of psychiatry. 1992 Oct;34(4):285.

9. Andrade C, Agarwal AK, Reddy MV. The practice of ECT in India: II. The practical administration of ECT. Indian Journal of Psychiatry. 1993 Apr;35(2):81.

10. Mudur G. Indian group seeks ban on use of electroconvulsive therapy without anaesthesia.

11. Bwalya GM, Srinivasan $V$, Wang $M$. Electroconvulsive therapy anesthesia practice 
patterns: results of a UK postal survey. The journal of ECT. 2011 Mar 1;27(1):81-5.

12. Dewald PA, Margolis NM, Weiner H. Vertebral fractures as a complication of electroconvulsive therapy. Journal of the American Medical Association. 1954 Mar 20;154(12):981-4.

13. Chanpattana $W$, Kramer BA, Kunigiri G, Gangadhar $B N$, Kitphati R, Andrade C. A survey of the practice of electroconvulsive therapy in Asia. The journal of ECT. 2010 Mar 1;26(1):5-10.

14. Nelson AI. A national survey of electroconvulsive therapy use in the Russian Federation. The journal of ECT. 2005 Sep 1;21(3):151-7.

15. Chanpattana W, Kunigiri G, Kramer BA, Gangadhar $B N$. Survey of the practice of electroconvulsive therapy in teaching hospitals in India. The journal of ECT. 2005 Jun 1;21(2):100-4.

16. Gosselin C, Graf P, Milev R, Delva N, Lawson JS, Enns M, Gilron I, Martin B, Chan P, Patry S, Jewell M. Delivery of electroconvulsive therapy in Canada: a first national survey report on devices and technique. The journal of ECT. 2013 Sep 1;29(3):22530.

17. Chanpattana W. A questionnaire survey of ECT practice in Australia. The journal of ECT. 2007 Jun 1;23(2):89-92.

18. Subedi S, Aich TK, Sharma N. Use of ect in nepal: A one year study from the country's largest psychiatric facility. Journal of Clinical and Diagnostic Research: JCDR. 2016 Feb;10(2):VC01.

19. Sherchan S, Joshi D. Clinical and demographic profile of patients receiving ECT in an institute. Journal of Nepal Health Research Council. 2009;7(1):10-3. 